\title{
Control of appetitive and aversive taste-reactivity responses by an auditory conditioned stimulus in a devaluation task: A FOS and behavioral analysis
}

\author{
Erin C. Kerfoot, ${ }^{1}$ Isha Agarwal, ${ }^{2}$ Hongjoo J. Lee, and Peter C. Holland ${ }^{3}$ \\ Johns Hopkins University, Baltimore, Maryland 21218, USA
}

\begin{abstract}
Through associative learning, cues for biologically significant reinforcers such as food may gain access to mental representations of those reinforcers. Here, we used devaluation procedures, behavioral assessment of hedonic taste-reactivity responses, and measurement of immediate-early gene (IEG) expression to show that a cue for food engages behavior and brain activity related to sensory and hedonic processing of that food. Rats first received a tone paired with intraoral infusion of sucrose. Then, in the absence of the tone, the value of sucrose was reduced (Devalue group) by pairing sucrose with lithium chloride (LiCl), or maintained (Maintain group) by presenting sucrose and $\mathrm{LiCl}$ unpaired. Finally, taste-reactivity responses to the tone were assessed in the absence of sucrose. Devalue rats showed high levels of aversive responses and minimal appetitive responses, whereas Maintain rats exhibited substantial appetitive responding but little aversive responding. Control rats that had not received tone-sucrose pairings did not display either class of behaviors. Devalue rats showed greater FOS expression than Maintain rats in several brain regions implicated in devaluation task performance and the display of aversive responses, including the basolateral amygdala, orbitofrontal cortex, gustatory cortex (GC), and the posterior accumbens shell (ACBs), whereas the opposite pattern was found in the anterior ACBs. Both Devalue and Maintain rats showed greater FOS expression than control rats in amygdala central nucleus, GC, and both subregions of ACBs. Thus, through associative learning, auditory cues for food gained access to neural processing in several brain regions importantly involved in the processing of taste memory information.
\end{abstract}

Reinforcer devaluation procedures are often used to assess cues' ability to guide behavior based on their access to a representation of the current incentive value of the reinforcer. For example, after tone-food pairings, the establishment of an aversion to the food reinforcer results in the spontaneous reduction of rats' learned food-cup approach responses to the tone, when it is presented later in the absence of food (Holland and Straub 1979). Thus, the rats' response to the tone is sensitive to changes in reinforcer value, despite no explicit experience of the tone together with the devalued reinforcer. Recent studies (for review, see Holland and Gallagher 2004) showed that this sensitivity of previously learned behaviors to subsequent alterations in reinforcer value demands function of a brain system that includes the basolateral amygdala (BLA) and the lateral orbitofrontal cortex (OFC).

Previous devaluation studies examined changes in performance of learned responses preparatory to the receipt of food, such as food-cup approach. Here, we considered whether a learned cue for food would provoke consummatory responses that reflect the current sensory-hedonic aspects of food. In the absence of food itself, would a food cue provoke "liking" or "disgust" responses appropriate to the current value of that food, as if the rat "tasted" the absent food? Rats show distinguishable appetitive and aversive orofacial responses to palatable and unpalatable flavors, respectively (e.g., Berridge 2000). Furthermore, when a palatable flavor is paired with the toxin lithium chloride

Present addresses: 'Psychology Department, University of Virginia, 102 Gilmer Hall, Charlottesville, VA 22904-4400, USA; ${ }^{2}$ Harvard College, Harvard University, University Hall, Cambridge, MA 02138, USA.

${ }^{3}$ Corresponding author.

E-mail pch@jhu.edu; fax (410) 516-0494.

Article is online at http://www.learnmem.org/cgi/doi/10.1101/lm.627007.
(LiCl), the form of these taste-reactivity responses shifts from appetitive to aversive (e.g., Berridge et al. 1981).

The results of some earlier studies suggested that the hedonic properties of foods could be transferred to cues by associative learning. For example, Delamater et al. (1986) found that auditory cues paired with sucrose or quinine later could provoke appetitive or aversive taste-reactivity responses, respectively, when those cues were presented while unflavored water was infused into the rats' oral cavities. Delamater et al. (1986) interpreted these data as indicating that the tones had acquired the hedonic properties of sucrose and quinine, but as Berridge and Schulkin (1989) noted, they might, instead, reflect simple stimulus-response (S-R) learning, by which the behavior pattern initially controlled by the reinforcer was transferred to the control of the auditory cues.

Berridge and Schulkin (1989) used a reinforcer revaluation procedure to demonstrate the acquisition of control of hedonic taste-reactivity responses by learned cues under circumstances that made an S-R account unlikely. They exploited the observation that under normal conditions rats find concentrated salt solutions aversive and display aversive taste-reactivity responses, but when sodium-depleted, they prefer such solutions and display appetitive taste-reactivity responses (Berridge and Schulkin 1989). In their experiment, rats first received pairings (in simultaneous compounds) of a neutral flavor cue (weak acetic acid or quinine) with a concentrated $(0.39 \mathrm{M}) \mathrm{NaCl}$ solution, which elicited aversive taste-reactivity responses, and the other neutral flavor with a sucrose solution. Next, the rats were made sodium deficient by injection of furosemide, and finally, responding to the flavor cues alone (acetic acid and quinine) was assessed in the absence of salt. In test, the rats displayed appetitive tastereactivity responses to the flavor cue that had previously been paired with $\mathrm{NaCl}$, consistent with the change in rats' taste- 
reactivity responses to concentrated $\mathrm{NaCl}$ itself. Notably, at the time of the original cue- $\mathrm{NaCl}$ pairings, only aversive tastereactivity responses occurred. Thus, only aversive responses could have been acquired by S-R learning. Instead, in testing, the flavor cue accessed the current (positive) hedonic significance of the $\mathrm{NaCl}$ reinforcer with which it had been paired.

In the present study, we used procedures analogous to those of Berridge and Schulkin (1989) to examine changes in tastereactivity responding to cues for sucrose after the value of sucrose was reduced by pairing it with illness (Table 1). The rats first received either paired or unpaired presentations of a tone and intraoral infusion of sucrose. Then, in the absence of the tone, they received infusions of sucrose either paired (Devalue) or unpaired (Maintain) with LiCl. Finally, we examined taste-reactivity responding of the rats to the tone alone, in the absence of sucrose (unflavored water was delivered to provide a substrate for oral responses). If the tone accessed the rats' current hedonic evaluation of sucrose, it would evoke appetitive responses in the Maintain rats and aversive responses in the Devalue rats (which would have never displayed aversive responses to the tone previously).

After behavioral testing, we examined FOS expression to evaluate learning-dependent neural activity. We anticipated that rats in the Devalue and Maintain conditions would differ in their FOS expression in BLA and OFC, regions known to be critical to devaluation performance, and in subregions of the nucleus accumbens (ACB) known to be involved in the expression of taste reactivity responses (Reynolds and Berridge 2002). Furthermore, if the tone activated sensory aspects of the sucrose, conditioningdependent activity might be observed in gustatory cortex (GC). Finally, we evaluated FOS in amygdala central nucleus (CeA), a region implicated in both tone-food and taste-aversion learning but not devaluation performance (Hatfield et al. 1996; Lamprecht and Dudai 2000), and the feeding-related lateral hypothalamus (LH).

\section{Results}

\section{Behavior: Acquisition and devaluation}

Table 2 shows responding in the training and devaluation sessions. Over the course of the training session, the rats that received paired presentations of tone and sucrose (groups Maintain and Devalue) acquired appetitive responses to the tone alone, whereas the rats in the two control groups, which received only tone presentations in that session (but sucrose alone in the next session) did not. All groups responded similarly to sucrose infusions in the devaluation session, although the control rats showed slightly more appetitive responding in the pre-sucrose intervals than the rats in groups Maintain and Devalue. This difference might reflect the common observation (e.g., Durlach 1983) of greater context conditioning after unsignaled than signaled reinforcer presentations. Very few aversive responses were observed in any of these sessions, although these responses were somewhat more common among the control rats.

Table 1. Outline of behavioral procedures

\begin{tabular}{llll}
\hline Group & \multicolumn{1}{c}{ Training } & Devaluation & \multicolumn{1}{c}{ Test } \\
\hline Devalue & Tone $\rightarrow$ sucrose & Sucrose $\rightarrow \mathrm{LiCl}$ & Tone $\rightarrow$ water \\
Maintain & Tone $\rightarrow$ sucrose & Sucrose; $\mathrm{LiCl}$ & Tone $\rightarrow$ water \\
CTL-d & Tone; sucrose & Sucrose $\rightarrow \mathrm{LiCl}$ & Tone $\rightarrow$ water \\
CTL-m & Tone; sucrose & Sucrose; $\mathrm{LiCl}$ & Tone $\rightarrow$ water
\end{tabular}

The right arrow $(\rightarrow)$ signifies paired presentations. On the training day, the rats in groups CTL-d and CTL-m received separate tone and sucrose sessions, 2-3 $\mathrm{h}$ apart. On the devaluation day, the rats in groups Maintain and CTL-m received lithium chloride ( $\mathrm{LiCl}$ ) injections 6-8 $\mathrm{h}$ after the sucrose session.
A training contingency (tone paired or unpaired with sucrose) $\times$ devaluation contingency (sucrose to be devalued or maintained) $\times$ observation interval (pre-tone, tone-alone, tone + sucrose/nothing, or post-tone) ANOVA of appetitive response scores in the first training session showed significant effects of training contingency, $F_{(1,18)}=29.16, P<0.0001$, and observation interval, $F_{(3,54)}=32.86, P<0.001$, and a significant interaction of those two variables, $F_{(3,54)}=28.59, P<0.001$. Subsequent ANOVAs for each observation interval separately showed no significant main effects or interactions for the pretone interval, $P$ s $>0.1136$, but reliable effects of training contingency during each of the remaining observation intervals, including the tone-alone interval, $F_{(1,18)}=9.23, P=0.0071$; other $F s>22.92, P s<0.0002$. There were no significant effects of subsequent devaluation contingency or that factor's interaction with training contingency, $F_{(1,18)} \mathrm{s}<2.03, P \mathrm{~s}>0.1722$. The paucity of aversive responses and severe violations of heterogeneity of variance assumptions $(P \mathrm{~s}<0.0001)$ made ANOVAs for that measure inappropriate. Despite the very low levels of aversive responding, distribution-free median tests showed significantly more aversive responding among the unpaired control rats than the paired rats during the tone and post-tone periods, $\mathrm{X}^{2}(1) \mathrm{s}>4.42, P \mathrm{~s}<0.0354$. Note that during these intervals, the rats in the paired groups displayed high levels of appetitive responses, which might have competed with the display of any very-low-baseline aversive responses.

ANOVA of appetitive behaviors of the control rats in their second training session (which included only sucrose delivery) showed a significant effect of observation interval (pre-sucrose, sucrose, or post-sucrose), but no effect of subsequent devaluation or interaction of those variables, $F \mathrm{~s}<1, P \mathrm{~s}>0.8600$. For aversive responses, a Freidman distribution-free test showed no effect of observation interval in that session, $\mathrm{X}^{2}(2)=0.67, P=0.7165$, and a medians test showed no effects of subsequent devaluation, $\mathrm{X}^{2}(1) \mathrm{s} \leq 2$, s $>0.1572$.

A training contingency $\times$ devaluation contingency $\times$ observation interval (pre, during, and post-sucrose delivery) ANOVA of appetitive behaviors during the devaluation session showed a significant effect of observation interval, but no significant effects or interactions of the remaining variables, $P$ s $>0.1580$. Median tests showed significantly more aversive responding in tone-sucrose unpaired rats than in paired rats during the pre-sucrose periods, $\mathrm{X}^{2}(1)=5.32, P=0.0210$, but not the other observation intervals, $P \mathrm{~s}>0.0704$.

\section{Behavior: Test session}

Figure 1 shows the behavioral results of the test session. Rats in group Maintain, which received tone-sucrose pairings, but sucrose and LiCl unpaired, displayed high levels of appetitive responding (Fig. 1A) during the tone-alone, tone + water, and posttone observation intervals, despite the absence of sucrose presentations during the test session. In contrast, the rats in group Devalue, which received tone-sucrose pairings but later had sucrose paired with $\mathrm{LiCl}$ (in the absence of the tone), showed no more appetitive responses than control rats that had never received tone-sucrose pairings. Thus, the effects of post-training devaluation of the sucrose reinforcer on responding to the tone were complete. Furthermore, the rats in group Devalue displayed significantly more aversive responses (Fig. 1B) after tone presentations than rats in any of the other groups. Notably, the rats in group Devalue had never previously displayed aversive responses to the tone, or at any time while they were in the experimental context. Instead, those rats had experienced illness in their home cages after exposure to sucrose alone in that context. Thus, learned responding controlled by the tone was sensitive to 
Table 2. Taste-reactivity responses during training and devaluation sessions

\begin{tabular}{|c|c|c|c|c|}
\hline & \multicolumn{4}{|c|}{ Appetitive responses } \\
\hline & \multicolumn{4}{|c|}{ Group } \\
\hline & Maintain & Devalue & CTL-m & CTL-d \\
\hline \multicolumn{5}{|l|}{ Training session 1} \\
\hline Pre-tone & $1.88 \pm 0.20$ & $2.10 \pm 0.45$ & $1.05 \pm 0.27$ & $1.72 \pm 0.53$ \\
\hline Tone alone & $2.89 \pm 0.30$ & $2.49 \pm 0.45$ & $1.07 \pm 0.25$ & $1.83 \pm 0.66$ \\
\hline Tone + sucrose or & $3.96 \pm 0.35$ & $3.63 \pm 0.60$ & - & - \\
\hline Tone alone & - & - & $1.34 \pm 0.19$ & $1.82 \pm 0.46$ \\
\hline \multirow{2}{*}{\multicolumn{5}{|c|}{$\begin{array}{l}\text { Post-tone } \\
\text { Training session } 2\end{array}$}} \\
\hline & & & & \\
\hline Pre-sucrose & - & - & $2.16 \pm 0.50$ & $2.08 \pm 0.48$ \\
\hline Sucrose & - & - & $2.77 \pm 0.31$ & $2.75 \pm 0.70$ \\
\hline Post-sucrose & - & - & $7.29 \pm 0.88$ & $7.63 \pm 1.20$ \\
\hline \multicolumn{5}{|l|}{ Devaluation session } \\
\hline Pre-sucrose & $2.25 \pm 0.37$ & $1.33 \pm 0.27$ & $2.25 \pm 0.56$ & $2.92 \pm 1.00$ \\
\hline Sucrose & $4.06 \pm 0.57$ & $3.30 \pm 0.32$ & $3.63 \pm 0.75$ & $4.00 \pm 0.71$ \\
\hline \multirow[t]{3}{*}{ Post-sucrose } & $5.78 \pm 0.69$ & $4.67 \pm 0.26$ & $4.99 \pm 0.83$ & $4.97 \pm 0.66$ \\
\hline & \multicolumn{4}{|c|}{ Aversive behaviors } \\
\hline & Maintain & Devalue & CTL-m & CTL-d \\
\hline \multicolumn{5}{|l|}{ Training session 1} \\
\hline Pre-tone & $0.01 \pm 0.02$ & $0.02 \pm 0.01$ & $0.01 \pm 0.01$ & $0.07 \pm 0.04$ \\
\hline Tone alone & $0.02 \pm 0.02$ & $0.01 \pm 0.01$ & $0.04 \pm 0.03$ & $0.08 \pm 0.03$ \\
\hline Tone + sucrose or & $0.01 \pm 0.01$ & $0.01 \pm 0.01$ & - & - \\
\hline Tone alone & - & - & $0.02 \pm 0.02$ & $0.03 \pm 0.04$ \\
\hline Post-tone & $0.01 \pm 0.01$ & $0.00 \pm 0.00$ & $0.02 \pm 0.01$ & $0.11 \pm 0.05$ \\
\hline \multicolumn{5}{|l|}{ Training session 2} \\
\hline Pre-sucrose & - & - & $0.03 \pm 0.03$ & $0.09 \pm 0.04$ \\
\hline Sucrose & - & - & $0.08 \pm 0.06$ & $0.07 \pm 0.05$ \\
\hline Post-sucrose & - & - & $0.05 \pm 0.02$ & $0.02 \pm 0.01$ \\
\hline \multicolumn{5}{|l|}{ Devaluation session } \\
\hline Pre-sucrose & $0.00 \pm 0.00$ & $0.01 \pm 0.01$ & $0.03 \pm 0.03$ & $0.20 \pm 0.09$ \\
\hline Sucrose & $0.00 \pm 0.00$ & $0.08 \pm 0.06$ & $0.10 \pm 0.06$ & $0.13 \pm 0.09$ \\
\hline Post-sucrose & $0.01 \pm 0.01$ & $0.00 \pm 0.00$ & $0.04 \pm 0.03$ & $0.08 \pm 0.04$ \\
\hline
\end{tabular}

Entries are mean \pm SEM observations per 10-sec period (see text for scoring details). A dash (-) signifies events not received by rats in the group specified. The rats in groups Maintain and Devalue received tone-sucrose pairings in Training Session 1, whereas the rats in groups CTL-d and CTL-m received tone-alone presentations in that session and sucrose-alone presentations in Training Session 2. The rats in groups Maintain and Devalue did not receive Training Session 2.

changes in the rats' hedonic evaluation of the sucrose. Finally, neither appetitive nor aversive taste reactivity responses occurred with appreciable frequency in the control rats, which received tone and sucrose unpaired in training. This observation shows that the test behavior observed in groups Maintain and Devalue was the consequence of the associative learning contingencies arranged in the training session.

These adjustments to the form of taste-reactivity responses were observed in response to the tone alone but were more obvious when the tone was accompanied by a substrate (unflavored water), and, especially, after the termination of the delivery of tone and water. Notably, a comparable pattern of taste-reactivity responding is frequently observed to simple presentation of hedonically valued liquids, with maximum levels of that responding occurring long after the initial flavor delivery and continuing after flavor termination (Berridge 2000).

A training contingency $\times$ devaluation contingency $\times$ observation interval ANOVA of appetitive responding in the test session showed significant effects of all three variables, Fs $>8.21$, $P s<0.0076$, and interactions of the training contingency with both the devaluation contingency, $F_{(1,18)}=6.11, P=0.0236$, and the observation interval, $F_{(3,54)}=4.18, P=0.0099$. Subsequent training $\times$ devaluation contingency ANOVAs for each observation interval showed no significant effects or interactions in the pre-tone periods, $F \mathrm{~s}<3.72, P \mathrm{~s}>0.0699$, a significant effect of de- valuation contingency in the tone-alone periods, $F_{(1,18)}=4.47$, $P=0.0487$, and significant effects of training contingency in the tone + water periods, $F_{(1,18)}=7.70, P=0.0125$. ANOVA of posttone appetitive responding showed significant main effects of both training, $F_{(1,18)}=15.32, P=0.0010$, and devaluation, $F_{(1,18)}=20.93, P=0.0002$, contingencies, and their interaction, $F_{(1,18)}=10.53, P=0.0045$. Individual contrasts showed that responding in group Maintain was significantly higher than responding in each other group during each of the observation intervals, $P \mathrm{~s}<0.0400$, except the pre-tone intervals, $P \mathrm{~s}>0.0955$. No other individual comparison among the groups was significant, $P$ s $>0.4048$.

A comparable ANOVA of aversive responding showed significant main effects of devaluation contingency, $F_{(1,18)}=10.87$, $P=0.0040$, and observation interval, $F_{(3,54)}=9.83, P<0.0001$, as well as significant interactions between training and devaluation contingencies, $F_{(1,18)}=6.86, P=0.0174$, and among all three variables, $F_{(3,54)}=6.94, P=0.0005$. Separate training $\times$ devaluation contingency ANOVAs for aversive responding in each observation interval showed a significant effect of devaluation contingency in each interval, $F_{(1,18)} \mathrm{s}>7.55, P \mathrm{~s}<0.0133$. The training $\times$ devaluation contingency interaction was significant only during the post-tone interval, $F_{(1,18)}=15.97, P=0.0008$. Planned individual contrasts showed significantly more aversive responses in group Devalue than in any other groups during the 


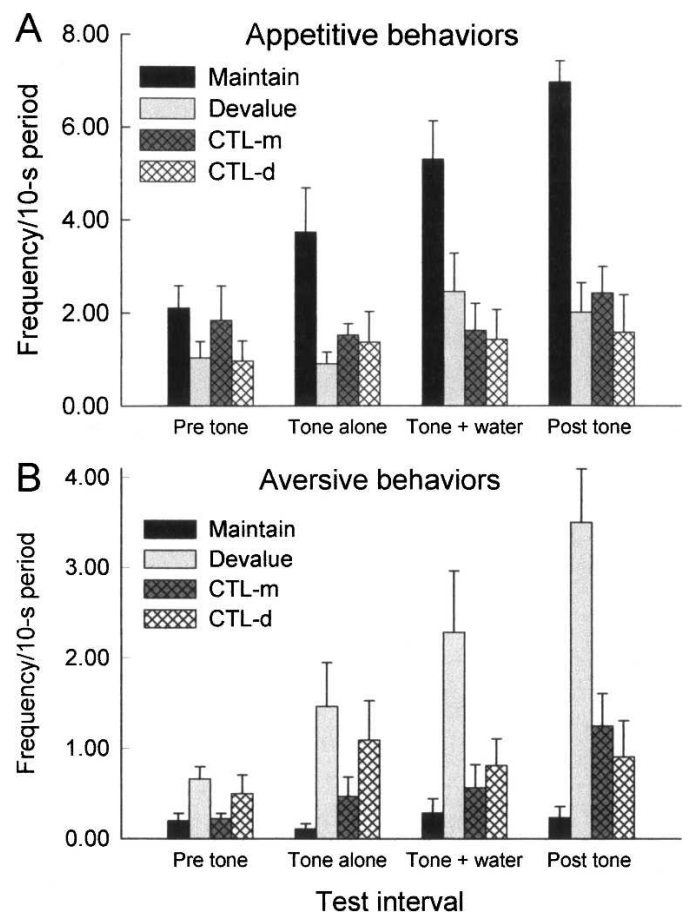

Figure 1. Mean \pm SEM taste-reactivity responses in the test session. Each trial comprised a 10-sec pre-tone period, $10 \mathrm{sec}$ of tone alone, $5 \mathrm{sec}$ of tone accompanied by deionized water, and a $10 \mathrm{sec}$ post-tone interval. Rats in group Maintain had received tone $\rightarrow$ sucrose pairings followed by sucrose and toxin unpaired. Rats in group Devalue had received tone $\rightarrow$ sucrose pairings followed by sucrose-toxin pairings. Rats in the two control (CTL) groups had received tone and sucrose unpaired followed by sucrose either paired ( $d$, devalue) or unpaired ( $m$, maintain) with toxin. (A) Appetitive responses; (B) aversive responses.

post-tone interval, $P s<0.0025$, and the tone + water interval, $P$ s $<0.0485$, and significantly more than in group Maintain during the tone alone, $P=0.0076$, and pre-CS, $P=0.0489$, periods. No other comparisons were significant.

\section{Behavior: Sucrose test}

To evaluate the effects of the devaluation treatments on taste responses to sucrose itself, two rats in group Maintain and two rats in group Devalue received a second test session, which included sucrose-alone infusions. In the post-sucrose observation intervals, the two rats in group Maintain showed 11.1 and 13.2 appetitive and 1.2 and 0.06 aversive responses per 10 -sec interval, whereas the two rats in group Devalue showed 1.6 and 3.4 appetitive and 10.4 and 7.1 aversive responses. The distribution of subcategories within each class of responses was similar to that observed in the preceding test.

\section{FOS immunocytochemistry}

Components of brain circuits previously identified as important for the display of devaluation effects showed differential FOS expression as a function of training and devaluation treatments (Fig. 2). In BLA, OFC, and GC (Fig. 2A), FOS expression was greater in group Devalue than in group Maintain or group Control, and it was greater in group Maintain than in group Control in GC. A similar pattern was observed in the posterior portions of the ACB shell (Fig. 2B), with greater FOS expression in group Devalue than in either groups Maintain or Control. In contrast, in the anterior portions of the ACB shell, FOS expression was greater in group Maintain than in either group Devalue or group Control. In addition, FOS expression in CeA was greater in the two groups that received paired presentations of tone and sucrose than in the unpaired control rats. Finally, there were no significant differences among the groups in FOS expression in LH or in either of the subregions of the ACB core.

One-way ANOVAs showed significant effects of group (Maintain, Devalue, or Control) on FOS counts/area in BLA, $F_{(2,11)}=9.16, P=0.0046, \mathrm{GC}, F_{(2,11)}=9.53, P=0.0040$, and OFC, $F_{(2,11)}=11.82, P=0.0018$. Individual comparisons showed that FOS expression was greater in group Devalue than in either group Control or Maintain for all three of these regions, $P \mathrm{~s}<0.0370$, and FOS was greater in group Maintain than in group Control for GC, $P=0.0370$. The apparent numerical differences between group Maintain and group Control in BLA and OFC were not significant, $P \mathrm{~S}=0.065$ and $P=0.144$, respectively. A similar ANOVA for CeA also showed a significant effect of group, $F_{(2,11)}=4.38, P=0.0400$ (mean \pm SEM for FOS + cells/ $\mathrm{mm}^{2}=187.0 \pm 16.8,182.2 \pm 13.6$, and $115.2 \pm 21.8$ in groups Maintain, Devalue, and CTL, respectively). Individual comparisons showed greater FOS in CeA in both groups Maintain and Devalue than in group Control, $P \mathrm{~s}<0.0289$. There was no difference in FOS in LH among the groups $(250.2 \pm 36.9,276.7 \pm 20.4$, $263.8 \pm 16.7$, respectively), $F_{(1,11)}=0.28, P=0.7578$.

A group $\times$ subregion (anterior or posterior) ANOVA of FOS in the ACB shell showed only a significant interaction, $F_{(1,11)}=4.81, P=0.0315$. Individual comparisons showed that in the anterior shell, FOS was greater in group Maintain than in either group Control, $P=0.0051$, or group Devalue, $P=0.0490$, but in the posterior shell, FOS was greater in group Devalue than in either groups Control, $P=0.0023$, or Maintain, $P=0.0050$. In addition, in group Devalue, FOS expression was significantly greater in the posterior shell than in the anterior shell, $P=0.0291$. Although in group Maintain, FOS expression appeared numerically greater in the anterior than in the posterior shell, that difference did not approach statistical significance, $P=0.1163$. A comparable ANOVA for the ACB core showed only a marginally significant effect of subregion, $F_{(1,11)}=4.63$, $P=0.0545$; other $P$ s $>0.2001$. The marginal effect of subregion
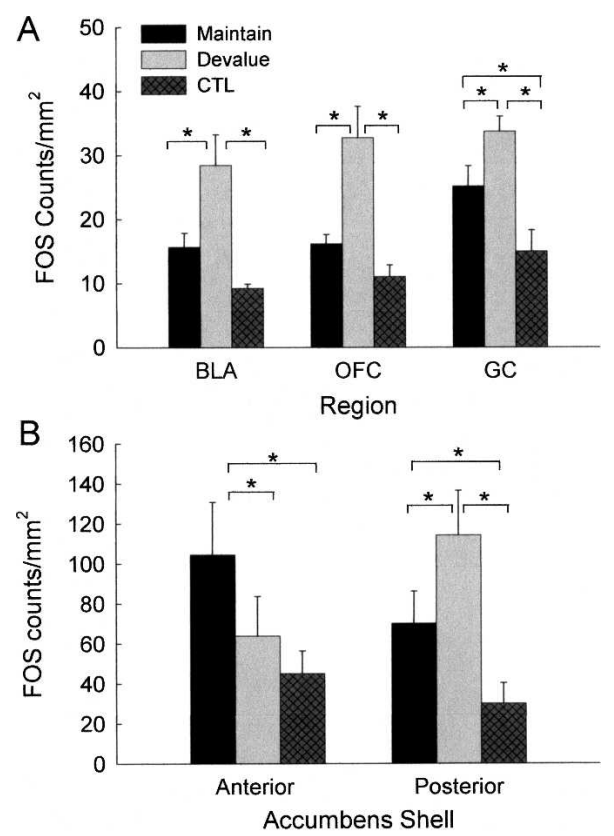

Figure 2. Mean \pm SEM FOS-immunoreactive cells $/ \mathrm{mm}^{2}$ in $(A)$ basolateral amygdala (BLA), orbitofrontal cortex (OFC), gustatory cortex (GC) and $(B)$ nucleus accumbens shell. Starred brackets indicate significant $(P<0.05)$ differences. 
reflected a trend toward greater FOS expression in the posterior core $(110.9 \pm 38.1,110.7 \pm 21.7$, and $182.6 \pm 92.6$ FOS counts/ $\mathrm{mm}^{2}$ in groups Maintain, Devalue, and CTL) than in the anterior core $(86.8 \pm 21.7,98.9 \pm 20.7$, and $22.8 \pm 27.7)$.

\section{Discussion}

Post-training devaluation of a sucrose reinforcer reduced previously conditioned appetitive taste-reactivity responses controlled by a tone cue, to control levels. In addition, after devaluation, the tone controlled the display of aversive responses, which had never previously occurred in its presence. Thus, in test the tone cue evoked responses appropriate to the current hedonic evaluation of the sucrose, not the value of sucrose when the tone had been paired with it. Indeed, because there was only a single pairing of sucrose deliveries with illness in the devaluation phase, and sucrose was not presented in test, the rats had never even experienced devalued sucrose before. Nevertheless, they were able to combine initial tone-sucrose information with the subsequently acquired changes in the hedonic value of sucrose to perform responses appropriate to that updated value in the presence of the tone alone. These findings extend the observations of Berridge and Schulkin (1989), who found a spontaneous shift from aversive to appetitive responses when the value of a salt solution was increased by manipulating a motivational state, by showing a complementary shift from appetitive to aversive responses when the value of sucrose was reduced by an associative manipulation, taste aversion learning.

It is notable that the devaluation effects observed here were essentially complete, substantially larger than those usually reported for more typical measures of Pavlovian or operant conditioned responses (e.g., Holland and Straub 1979; Colwill and Rescorla 1985; Hatfield et al. 1996; Pickens et al. 2003), and in a previously reported study that used taste-reactivity measures without oral cannulation (Holland 1990). Although it might be anticipated that evaluative, taste-reactivity responses would be especially sensitive to changes in reinforcer value, Colwill and Rescorla (1990) found that devaluation effects on operant lever press responding were also complete when the sucrose reinforcer had been delivered intraorally throughout lever-press training and the sucrose-LiCl devaluation experience. Thus, the use of intraoral reinforcer delivery may provide an especially sensitive devaluation procedure ideal for examining brain function.

The patterns of FOS expression observed after presentation of the tone in the test session provide information about how, through associative learning, predictive cues can activate brain systems involved in the processing of information about current events and upcoming reinforcers, especially taste memory information. First, rats that had received tone-sucrose pairings in training (groups Maintain and Devalue) displayed more FOS expression in CeA than rats that had received tone and sucrose unpaired, regardless of the subsequent fate of sucrose. This finding is comparable to Lee et al.'s (2005) observation of conditioning-dependent FOS expression in CeA after tone-food pairings in more traditional settings, in which food was delivered to a cup rather than intraorally. The lack of a difference in CeA FOS expression between groups Devalue and Maintain, although consistent with observations that lesions of CeA have no effect on devaluation task performance (Hatfield et al. 1996), is notable nevertheless, because many studies have implicated CeA in taste aversion learning. Flavor and visceral information converge in $\mathrm{CeA}$, and presentation of either a novel taste or $\mathrm{LiCl}$ enhances FOS expression in CeA (e.g., Bernstein and Koh 2007). Furthermore, acquisition of flavor aversions is disrupted by infusion of protein synthesis inhibitors (Bahar et al. 2003) or fos antisense (Lamprecht and Dudai 1996) into CeA during a taste aversion learning trial. Similarly, Yamamoto (2007) found enhanced sensitivity of taste-selective cells in the parabrachial nucleus (PBN) when CeA was stimulated, an effect that also occurs after taste aversion learning. Findings such as these led Bernstein and Koh (2007) to suggest that CeA is especially involved in the enhanced processing of flavor CSs, which may be important for the formation of taste aversion memories. However, the lack of a difference between groups Maintain and Devalue in the present study, in which FOS expression was examined after tone presentations, and not sucrose itself, suggests that tone-sucrose conditioning did not give the tone access to any such changes in processing of the sucrose flavor memory that may have occurred in CeA as a result of flavor aversion learning in group Devalue. Recall that in our test, only the tone cue for sucrose, and not sucrose itself, was presented. Instead, as suggested by Lee et al. (2005), the conditioning-dependent FOS responses in CeA might reflect enhanced attentional processing of the tone itself as a result of tone-sucrose pairings, rather than associatively activated processing of a taste memory.

Second, consistent with the results of lesion studies (Hatfield et al. 1996; Gallagher et al. 1999), we found devaluationdependent differences in FOS expression in OFC and BLA, but (again) not in CeA. Notably, in both BLA and OFC, we found greater FOS expression in group Devalue than in group Maintain, as if arousal of a memory of the sucrose reinforcer led to greater neural activity in these regions after its association with illness. On the surface, this observation contrasts with the results of recent human imaging experiments, in which the blood oxygenation-level-dependent response in BLA and OFC to a visual cue for a particular food was reduced after devaluation of that food by selective satiation (Gottfried et al. 2003). However, in those experiments, selective satiation is likely to have reduced the positive value of the sated food without necessarily making it aversive, as in our experiment, in which sucrose was devalued by pairing it with illness.

Although in our study, group Maintain showed numerically more FOS expression in BLA and OFC than group CTL, in which the tone had not been paired with sucrose, these differences were not statistically significant ( $P$ s $=0.065$ and 0.144 , respectively). It is possible that neurons in these regions coded only aversive information about the expected reinforcer, and thus the FOS response was only significantly greater than the control response in group Devalue. However, we think it is more likely that BLA and OFC each coded both positive and negative incentive values of the sucrose taste memory, and that the tone cue accessed that value. Consistent with this view, electrophysiological studies (e.g., Schoenbaum, et al. 1998, 1999) of rats engaged in simple conditioning tasks (i.e., not devaluation procedures) show that neurons in these regions respond selectively to signals for appetitive or aversive flavors, and to those flavors themselves. Interestingly, several studies in which BLA coding of food or shockassociated cues was examined, using either electrophysiological (Paton et al. 2006) or immediate early gene expression (McDannald et al. 2006) techniques, suggest that these kinds of appetitive and aversive information are coded by separate, but spatially intermixed subpopulations of neurons within the BLA. Hence, more refined analysis of neuronal responding in the present devaluation task would be of considerable interest.

Third, the topographically distinct patterns of FOS expression we observed in the ACB shell (but not ACB core) after the presentation of a signal for sucrose are especially interesting. Rats in group Maintain, which displayed appetitive taste-reactivity responses in test, showed more FOS expression in the anterior shell than in the posterior shell, whereas rats in group Devalue, which exhibited aversive taste-reactivity responses, showed more FOS in the posterior shell than in the anterior shell. These pat- 
terns are generally consistent with the results of Reynolds and Berridge (2002), who found that the frequency of normal appetitive taste-reactivity responses to sucrose was enhanced only by stimulation of the most anterior portions of the ACB shell, but aversive responses were enhanced more by stimulation of the posterior ACB shell. Thus, our data indicate that through associative learning, cues for food can gain access to circuits that are involved in the representation and expression of current hedonic information about that food.

Many researchers have suggested that, as a primary consequence of associative learning, cues may acquire motivational or hedonic characteristics of the reinforcers that they signal (e.g., Bindra 1978), and that these acquired characteristics mediate many examples of goal-directed behavior. However, as noted above, it is clear that in the present experiment the tone did not simply acquire the hedonic value of the sucrose with which it was paired. If that were the case, in test the tone would have controlled appetitive taste-reactivity responses in both groups Maintain and Devalue. Instead, the tone controlled hedonic responses appropriate to the current value of the sucrose, and thus must have accessed pre-evaluative processing of the absent sucrose (Toates 1986; Berridge 2001), which then guided behavior appropriate to the altered evaluation of sucrose.

An intriguing possibility is that the tone associatively activated perceptual processing of the absent sucrose. From this perspective, consistent with the observation of conditioningdependent FOS expression in GC in both groups Maintain and Devalue, the tone aroused the perception of sweetness, as if it made the water taste sweet. Because rats in group Maintain responded to sweet (sucrose itself) with appetitive taste-reactivity responses (as shown in the sucrose test), the tone alone would also elicit those responses. Similarly, because rats in group Devalue would respond to sweet with aversive responses, by associatively activating a perception of sweetness, the tone would provoke aversive responses in that group. Notably, although GC is not critical to basic functions of taste detection (e.g., Dunn and Everitt 1988), it has been shown to be importantly involved in many aspects of higher-level processing of taste memories (e.g., Braun et al. 1982; Rosenblum et al. 1995, 1997). Most relevant to the present study, Kiefer and Orr (1992) found that rats with GC lesions failed to replace appetitive taste-reactivity responses to a sweet flavor with aversive responses after flavor-illness pairings, even under circumstances under which those lesioned rats successfully learned to suppress overall consumption of that flavor. Thus, GC may play a special role in the sensory-hedonic revaluation of flavors after pairing with illness, perhaps via its extensive connections with both OFC and BLA (e.g., Allen et al. 1991; McDonald 1998; Shi and Cassell 1998), whose involvement in devaluation performance was just discussed. The present data indicate further that food-associated cues alone may instigate such processing of sensory-hedonic information by GC. Notably, as in BLA and OFC, Fos expression in GC was greater in group Devalue than in group Maintain. Thus, it is of interest to determine whether GC neurons code both appetitive and aversive properties of anticipated reinforcers in this context, or only some generic "significance" of the tone or the sucrose memory (Anderson et al. 2003; Small et al. 2003), and that this significance was enhanced by sucrose-illness pairings.

Converging evidence shows that associatively activated memories of important events may substitute for those events themselves in several functions (for review, see Holland 1990; Holland and Gallagher 2004). In the present study, a tone substituted for sucrose in the production of taste-reactivity responses appropriate to the current evaluation of that associated, but absent, sucrose. A large body of research shows that associatively activated taste memories may also participate in new learning about those physically absent tastes (for review, see Holland and Wheeler 2007). For example, in one study (Holland 1981), rats were first trained to associate one auditory/visual cue with one food flavor and another cue with another food flavor. Then they were made ill in the presence of one of the cues, but in the absence of either food flavor. Finally, a test of consumption of the foods in the absence of the auditory/visual cues showed that the rats had acquired an aversion to the food flavor whose auditory/visual partner had been paired with illness.

These and other related behavioral findings (Holland and Wheeler 2007), taken together with the patterns of FOS expression observed in the present experiment, are consistent with the notion that the associative activation of taste memories by relatively arbitrary cues such as tones and lights engages several brain systems involved in the formation, evaluation, modification, and use of those taste memories. These systems include amygdalarcortical networks known to be in involved in the representation, evaluation, and use of information about expected food reinforcers (e.g., Holland and Gallagher 2004; Pickens and Holland 2004), and ventral striatal systems known to be involved in the organization of information and action in motivated behavior systems (e.g., Reynolds and Berridge 2002). An important question that remains unanswered in the present study is whether the neurons in these systems that are activated by the tone as a consequence of tone-sucrose learning and (in some cases) subsequent devaluation of sucrose are the same neurons that would have been activated by valued or devalued sucrose itself. Although we assessed taste-reactivity responses to sucrose itself in a small subset of rats, we did not examine FOS in those rats. Nevertheless, assessment of FOS in those rats would have only been informative about whether the tone and sucrose itself activated neurons in similar locations within the monitored brain regions, across animals. Determining whether a cue activated the same neurons as its consequent, and identification of shared and unique aspects of neural activation by cues previously paired with now-devalued or value-maintained reinforcers, awaits use of more sophisticated imaging techniques that permit evaluation of selective neuronal activation by multiple events (e.g., Petrovich et al. 2005; Paton et al. 2006).

Our observation that tone-activated FOS expression in CeA, although dependent on tone-sucrose pairings, did not significantly differ as a function of post-training taste-aversion learning experience, suggests that associatively activated taste memories are not completely interchangeable with the tastes themselves. As noted earlier, taste and illness information converge in this region, and CeA is known to be important in many aspects of the learning and expression of taste aversions. It is of interest to determine the conditions (if any) under which associatively activated taste memories may instigate these aspects of taste processing, and likewise in other brain regions in which taste and visceral information are known to converge, and which have been implicated in taste aversion learning, such as PBN and area postrema (Yamamoto 2007), but which were not available for analysis in this study.

\section{Materials and Methods}

\section{Subjects}

Twenty-four experimentally naive 350-400-g male Long-Evans rats (Charles River Laboratories) were housed individually. The vivarium was climate controlled and illuminated from 6:00 a.m. to 8:00 p.m. Rats were allowed ad libitum access to food and water until $\sim 7-10 \mathrm{~d}$ post-operative, when their access to food was restricted to maintain their weights at $85 \%$ of baseline. 


\section{Surgical procedures}

Rats were anesthetized with isoflurane gas (Abbot Laboratories) and placed in a stereotaxic frame (Kopf Instruments). Aseptic precautions were taken to prevent bacterial infection. Surgical procedures similar to those described by Grill and Norgren (1978) were used to implant an intraoral cannula just anterolateral to the second maxillary molar on each side. Each intraoral cannula consisted of PE-100 polyethylene tubing (Fisher Scientific) flared at one end and fitted with a washer to hold it in place in the mouth. Each cannula was threaded under the skin to a 1/2-inch segment of 19-gauge hypodermic tubing that served as a connector for fluid delivery. This connector was cemented in place on the rat's skull with dental acrylic, secured with four skull screws.

\section{Postoperative care}

Immediately after the surgery, rats received an injection of buprenorphin $(0.03 \mathrm{mg} / \mathrm{kg})$ to ameliorate pain. Rats were given 10 d to recover post-surgery, with daily oral administration of 0.5 $\mathrm{mL}$ of cephalexin $(250 \mathrm{mg} / 5 \mathrm{~mL}$; Teva Pharmaceuticals) to prevent infection. After $10 \mathrm{~d}$, cephalexin was discontinued, and the rats were placed on their food-restriction diet. The cannulas were flushed daily with deionized water throughout the recovery and experimental periods. Two rats died prior to behavioral training.

\section{Apparatus}

The behavioral training apparatus consisted of a clear PVC (polyvinyl chloride) cylinder $(36.8 \mathrm{~cm}$ tall and $28 \mathrm{~cm}$ in diameter) mounted on top of a clear floor. The apparatus was elevated on a stand measuring $36.8 \mathrm{~cm}$ in height. A color CCD camera was placed underneath the clear floor so that the full range of the cylinder was in view and the rats could be seen from below. A speaker (Moisture Resistant Speaker System MX1; Radio Shack) that delivered a $1000-\mathrm{Hz}$ tone was placed in the front left corner of the floor just outside the cylinder. A syringe pump (Razel Scientific Instruments, Inc.) for delivery of liquids was positioned at the bottom of the stand.

\section{Behavioral training}

All rats received behavioral training consisting of conditioning, devaluation, and test phases (Table 1). At the beginning of each session, a length of PE-100 tubing extending from a 1-mL syringe in the pump was attached to the intraoral cannula connector on the rat's head. Separate syringes and lines were used for sucrose and water. In the conditioning phase, the rats in groups Maintain $(n=7)$ and Devalue $(n=7)$ each received 30 tone-sucrose pairing trials in a single 60-min session. Each trial comprised a 15 -sec $1000-\mathrm{Hz}$ tone, with $0.1 \mathrm{~mL}$ of $8 \%(\mathrm{w} / \mathrm{v})$ sucrose delivered during the last $5 \mathrm{sec}$ of tone presentation. Intertrial intervals (ITIs) varied between 1 and $2 \mathrm{~min}$. In this phase, the rats in group CTL $(n=8)$ received the same number of tone and sucrose presentations, but in separate sessions, separated by $2-3 \mathrm{~h}$. The first session included 30 15-sec tone trials, and the second session included 305 -sec sucrose trials. In the devaluation phase, all rats first received 15 $0.1-\mathrm{mL} 5$-sec sucrose infusions over the course of $30 \mathrm{~min}$. The rats in group Devalue and the four rats in group CTL-d were removed from the cylinder immediately after the last trial, injected with $0.6 \mathrm{M}$ lithium chloride ( $\mathrm{LiCl} ; 5 \mathrm{ml} / \mathrm{kg}$ ), and returned to their home cages. The rats in group Maintain and the four rats in group CTL-m were instead placed back in their home cages, allowed to rest for $6-8 \mathrm{~h}$, and then injected with $\mathrm{LiCl}$. Thus, all rats received the same presentations of sucrose and $\mathrm{LiCl}$, but only those in group Devalue and group CTL-d should form an aversion to sucrose. After a rest day, all rats received a single 15-min test session, which included eight 15 -sec tone presentations, in which deionized water was infused in the last $5 \mathrm{sec}$ of each tone.
The water delivery provided a substrate for the display of orofacial taste-reactivity responses.

To evaluate the effects of sucrose- $\mathrm{LiCl}$ pairings on tastereactivity responding to sucrose itself, two rats in each of the groups Devalue and Maintain received an additional 15-min test session the next day, which included eight 5-sec infusions of sucrose.

\section{Behavioral analysis}

Behavior in each phase was recorded, and tapes were scored for appetitive and aversive behavioral responses. Behavioral scoring was divided into pre-trial (10 sec before trial start), tone-alone (10 sec during tone before liquid presentation), tone + liquid (last 5 sec of tone presentation, while sucrose, no liquid, or water, depending on the group and session, was infused), and post-trial (10 sec after the end of the tone) periods. Tapes were viewed at $1 / 10$ normal speed. Behavioral categories were modeled after Berridge (2000). Owing to their relatively long bouts, appetitive taste-reactivity responses were scored using a time-sampling procedure in which a judgment of whether a target behavior was in progress was made at each observation. Observations were made each $1.25 \mathrm{sec}$ of slow-motion playback, timed by a metronome. Appetitive behaviors included licking paws and fast licking with tongue protrusion (rhythmic tongue movements from the midline). Aversive behaviors typically showed very short bout lengths, and thus were scored by recording the absolute number of occurrences within each 1.25-sec sampling interval. Aversive behaviors included slow licking (no tongue protrusion, teeth remain visible during the lick), head-shaking, face-wiping, and gaping.

\section{Histology}

Expression of FOS, the protein product of the immediate early gene $c$-fos, was evaluated in five rats in each of the groups Maintain and Devalue, and four rats in group CTL (two that received sucrose-LiCl pairings and two that received those events unpaired). These rats were sacrificed $75 \mathrm{~min}$ after the beginning of the first test session. Rats were deeply anesthetized with isoflurane gas and perfused with $0.9 \%$ saline followed by $4 \%$ cold paraformaldehyde in $0.1 \mathrm{M}$ phosphate buffer $(\mathrm{PB})$. Brains were removed and post-fixed in a cryoprotectant of $4 \%$ paraformaldehyde in $0.1 \mathrm{M} \mathrm{PB}$ and $12 \%$ sucrose overnight. Brains were then frozen with powdered dry ice, stored at $-80^{\circ} \mathrm{C}$, and sliced on a freezing microtome. Sections ( $30 \mu \mathrm{m}$, coronal) were collected in four series. One series was used for FOS immunoreactivity, one for nissl staining to verify anatomical locations of adjacent sections immunoreacted for FOS, and the remaining series were eventually discarded.

\section{FOS immunoreactivity}

Free-floating sections were first washed in $0.3 \% \mathrm{H}_{2} \mathrm{O}_{2}$ in $0.1 \mathrm{M}$ PBS (contains $0.9 \%$ saline) for $30 \mathrm{~min}$ to protect against endogenous peroxidase within the tissue. After several rinses in PBS, tissues were incubated for $2 \mathrm{~h}$ in PBS containing 0.3\% Triton X (PBST) and 3\% normal goat serum (Vector Laboratories). Sections were then incubated with the FOS antibody (1:5000 dilution; Oncogene Research Products) in PBST containing 3\% normal goat serum for $72 \mathrm{~h}$ at $4^{\circ} \mathrm{C}$. After the primary antibody incubation, sections were rinsed in PBS, incubated in biotinylated secondary antibody (1:250 dilution; Vector Laboratories) for $1 \mathrm{~h}$, rinsed in PBS, and then incubated in avidin-biotin conjugate (Vector Laboratories) for $1 \mathrm{~h}$. After several rinses in PBS, tissues were reacted using the Vector SG kit for peroxidase (Vector Laboratories). Tissues were rinsed again in PBS and mounted on superfrost slides. After drying thoroughly for at least $2 \mathrm{~d}$, slides were 
dehydrated in ascending concentrations of alcohol, defatted in xylene, and coverslipped with Permount.

\section{Analysis of FOS expression}

All analyses were conducted blind with respect to training condition. Images of the selected FOS stained sections and the adjacent thionin-stained sections were acquired using a MicroPublisher RTV camera (QImaging). Borders for each region were then drawn on the images of the thionin-stained sections and transferred to the FOS section images using Adobe Photoshop. Borders for CeA, LH, and ACB shell and core were based on Paxinos and Watson's (1997) atlas. CeA and LH sections were those at 1.80, 2.12, and 2.3 posterior to bregma; anterior ACB sections were those at 2.70 and $2.20 \mathrm{~mm}$ anterior to bregma; and posterior $\mathrm{ACB}$ sections were those 1.00 and 0.70 anterior to bregma. Borders for the remaining structures were based on Swanson's Rat Brain Atlas (Swanson 1993); levels 27, 28, and 29 for BLA; levels 5, 7, 8, and 9 for OFC; and levels 10, 11, and 13 for GC. Using an image analysis system (NIH Image 1.63), a threshold for background density was set for each defined region on the FOS section, and FOS-positive cells with a density that was at least two standard deviations above the background threshold were counted automatically. The number of counts was then divided by the area included by the defined region.

\section{Acknowledgment}

Portions of these data were presented at the 2004 meeting of the Society for Neuroscience (poster 206.10). This research was supported in part by NIH grants MH053667 and MH065879.

\section{References}

Allen, G.V., Saper, C.B., Hurley, K.M., and Cechetto, D.F. 1991 Organization of visceral and limbic connections in the insular cortex of the rat. J. Comp. Neurol. 311: 1-16.

Anderson, A.K., Christoff, K., Stappen, I., Panitz, D., Ghahremani, D.G., Glover, G., Gabrieli, J.D., and Sobel, N. 2003. Dissociated neural representations of intensity and valence in human olfaction. Nat. Neurosci. 6: 196-202.

Bahar, A., Samuel, A., Hazvi, S., and Dudai, Y. 2003. The amygdalar circuit that acquires taste aversion memory differs from the circuit that extinguished it. Eur. J. Neurosci. 17: 1527-1530.

Bernstein, I. and Koh, M.T. 2007. Molecular signaling during taste aversion learning. Chem. Senses 32: 99-103.

Berridge, K.C. 2000. Measuring hedonic impact in animals and infants: Microstructure of affective taste reactivity patterns. Neurosci. Biobehav. Rev. 24: 173-198.

Berridge, K.C. 2001. Reward learning: Reinforcement, incentives, and expectations. Psychol. Learn. Motiv. 40: 223-278.

Berridge, K.C. and Schulkin, J. 1989. Palatability shift of a salt-associated incentive during sodium depletion. Q. J. Exp. Psychol. B 41: 121-138.

Berridge, K.C., Grill, H.J., and Norgren, R. 1981. Relation of consummatory responses and preabsorptive insulin release to palatability and learned taste aversions. J. Comp. Physiol. Psychol. 95: $363-382$.

Bindra, D. 1978. How adaptive behavior is produced: A perceptual-motivational alternative to response-reinforcement. Behav. Brain Sci. 1: 41-91.

Braun, J.J., Lassiter, P.S., and Kiefer, S.W. 1982. The gustatory neocortex of the rat. Physiol. Psychol. 10: $13-45$

Colwill, R.M. and Rescorla, R.A. 1985. Post conditioning devaluation of a reinforcer affects instrumental responding. J. Exp. Psychol. Anim. Behav. Process. 11: 120-132.

Colwill, R.M. and Rescorla, R.A. 1990. Effect of reinforcer devaluation on discriminative control of instrumental behavior. J. Exp. Psychol. Anim. Behav. Process. 16: 40-47.

Delamater, A.R., LoLordo, V.M., and Berridge, K.C. 1986. Control of fluid palatability by exteroceptive Pavlovian signals. J. Exp. Psychol. Anim. Behav. Process. 12: 143-152.

Dunn, L.T. and Everitt, B.J. 1988. Double dissociations of the effects of amygdala and insular cortex lesions on conditioned taste aversion, passive avoidance and neophobia in the rat using the excitotoxin ibotenic acid. Behav. Neurosci. 102: 3-23.

Durlach, P.J. 1983. Effects of signaling intertrial unconditioned stimuli in autoshaping. J. Exp. Psychol. Anim. Behav. Process. 9: 374-379.

Gallagher, M., McMahan, R.W., and Schoenbaum, G. 1999. Orbitofrontal cortex and representation of incentive value in associative learning. J. Neurosci. 19: 6610-6614.

Gottfried, J.A., O'Doherty, J., and Dolan, R.J. 2003. Encoding predictive reward value in human amygdala and orbitofrontal cortex. Science 301: 1104-1107.

Grill, H.J. and Norgren, R. 1978. Chronically decerebrate rats demonstrate satiation but not bait shyness. Science 201: 267-270.

Hatfield, T., Han, J.-S., Conley, M., Gallagher, M., and Holland, P.C. 1996. Neurotoxic lesions of the basolateral, but not central, amygdala interfere with Pavlovian second order conditioning and reinforcer devaluation effects. J. Neurosci. 16: 5256-5265.

Holland, P.C. 1981. Acquisition of representation mediated conditioned food aversions. Learn. Motiv. 12: 1-18.

Holland, P.C. 1990. Event representation in Pavlovian conditioning: Image and action. Cognition 37: 105-131.

Holland, P.C. and Gallagher, M. 2004. Amygdala-frontal interactions and reward expectancy. Curr. Opin. Neurobiol. 14: 148-155.

Holland, P.C. and Straub, J.J. 1979. Differential effects of two ways of devaluing the unconditioned stimulus after Pavlovian appetitive conditioning. J. Exp. Psychol. Anim. Behav. Process. 5: 65-78.

Holland, P.C. and Wheeler, D.S. 2007. Representation-mediated food aversions. In Conditioned taste aversion: Behavioral and neural processes (eds. S. Reilly and T. Schachtman), (in press). Oxford University Press, Oxford.

Kiefer, S.W. and Orr, M.R. 1992. Taste avoidance, but not aversion, learning in rats lacking gustatory cortex. Behav. Neurosci. 106: $140-146$

Lamprecht, R. and Dudai, Y. 1996. Transient expression of c-Fos in rat amygdala during training is required for encoding conditioned taste aversion memory. Learn. Mem. 3: 31-41.

Lamprecht, R. and Dudai, Y. 2000. The amygdala in conditioned taste aversion: It's there but where. In The amygdala: $A$ functional analysis (ed. J.P. Aggleton), pp. 331-351. Oxford University Press, Oxford.

Lee, H.J., Groshek, F., Petrovich, G.D., Cantalini, J.P., Gallagher, M., and Holland, P.C. 2005. Role of amygdalo-nigral circuitry in conditioning of a visual stimulus paired with food. J. Neurosci. 25: $3881-3888$

McDannald, M.A., Petrovich, G.D., Lee, H.J., Haberman, R.P., Gallagher, M., and Holland, P.C. 2006. Distinct populations of basolateral amygdala neurons are activated by appetitive and aversive stimuli. Society for Neuroscience Abstracts 36: 373.10.

McDonald, A.J. 1998. Cortical pathways to the mammalian amygdala. Prog. Neurobiol. 55: 257-332.

Paton, J.J., Belova, M.A., Morrison, S.E., and Salzman, D. 2006. The primate amygdala represents the positive and negative value of visual stimuli during learning. Nature 439: 865-870.

Paxinos, G. and Watson, C. 1997. The rat brain in stereotaxic coordinates, 3d ed. Academic Press, San Diego.

Petrovich, G.D., Holland, P.C., and Gallagher, M. 2005. Amygdalar and prefrontal pathways to the lateral hypothalamus are activated by a learned cue that stimulates eating. J. Neurosci. 25: 8295-8302.

Pickens, C.L. and Holland, P.C. 2004. Conditioning and cognition. Neurosci. Biobehav. Rev. 28: 651-661.

Pickens, C.L., Saddoris, M.P., Setlow, B., Gallagher, M., Holland, P.C., and Shoenbaum, G. 2003. Different roles for orbitofrontal cortex and basolateral amygdala in a reinforcer devaluation task. J. Neurosci. 23: $11078-11084$

Reynolds, S.M. and Berridge, K.C. 2002. Positive and negative motivation in nucleus accumbens shell: Bivalent rostrocaudal gradients for GABA-elicited eating, taste "liking"/"disliking" reactions, place preference/avoidance, and fear. J. Neurosci. 22: 7308-7320.

Rosenblum, K., Schul, R., Meiri, N., Hadari, Y.R., Zick, Y., and Dudai, Y. 1995. Modulation of protein tyrosine phosphorylation in rat insular cortex after conditioned taste aversion learning. Proc. Natl. Acad. Sci. 92: 1157-1161.

Rosenblum, K., Berman, D.E., Hazvi, S., Lamprecht, R., and Dudai, Y. 1997. NMDA receptor and the tyrosine phosphorylation of its $2 B$ subunit in taste learning in the rat insular cortex. J. Neurosci. 17: $5129-5135$.

Schoenbaum, G., Chiba, A.A., and Gallagher, M. 1998. Orbitofrontal cortex and basolateral amygdala encode expected outcomes during learning. Nat. Neurosci. 1: 155-159.

Schoenbaum, G., Chiba, A.A., and Gallagher, M. 1999. Neural encoding in orbitofrontal cortex and basolateral amygdala during olfactory discrimination learning. J. Neurosci. 19: 1876-1884.

Shi, C.J. and Cassell, M.D. 1998. Cortical, thalamic, and amygdaloid 
connections of the anterior and posterior insular cortices. J. Comp. Neurol. 399: 440-468.

Small, D.M., Gregory, M.D., Mak, Y.E., Gitelman, D., Mesulam, M.M., and Parish, T. 2003. Dissociation of neural representation of intensity and affective valuation in human gustation. Neuron 39: 701-711.

Swanson, L.W. 1993. Brain maps: Computer graphics files (Version 1.0). Elsevier, Amsterdam.
Toates, F. 1986. Motivational systems. Cambridge University Press, New York.

Yamamoto, T. 2007. Brain regions responsible for the expression of conditioned taste aversion in rats. Chem. Senses 32: 105-109.

Received May 11, 2007; accepted in revised form July 11, 2007. 


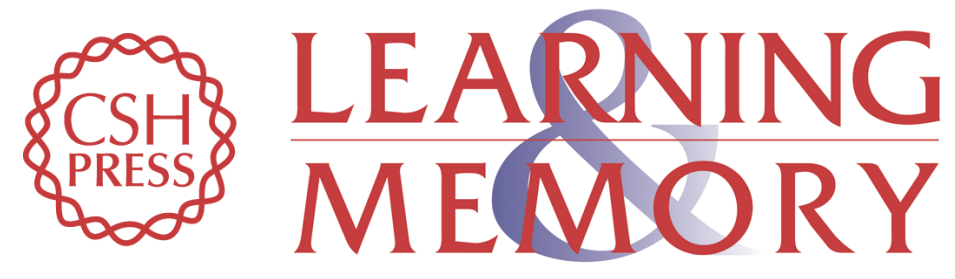

Control of appetitive and aversive taste-reactivity responses by an auditory conditioned stimulus in a devaluation task: A FOS and behavioral analysis

Erin C. Kerfoot, Isha Agarwal, Hongjoo J. Lee, et al.

Learn. Mem. 2007, 14:

Access the most recent version at doi:10.1101//m.627007

References This article cites 40 articles, 12 of which can be accessed free at: http://learnmem.cshlp.org/content/14/9/581.full.html\#ref-list-1

License

Email Alerting

Receive free email alerts when new articles cite this article - sign up in the box at the Service top right corner of the article or click here. 